\title{
Local Simulation Algorithms for Coulomb Interactions
}

\author{
A. C. Maggs and V. Rossetto \\ Laboratoire de Physico-Chime Théorique, ESPCI-CNRS, 10 rue Vauquelin, 75231 Paris Cedex 05, France.
}

(Dated: November 20, 2018)

\begin{abstract}
Long ranged electrostatic interactions are time consuming to calculate in molecular dynamics and MonteCarlo simulations. We introduce an algorithmic framework for simulating charged particles which modifies the dynamics so as to allow equilibration using a local Hamiltonian. The method introduces an auxiliary field with constrained dynamics so that the equilibrium distribution is determined by the Coulomb interaction. We demonstrate the efficiency of the method by simulating a simple, charged lattice gas.
\end{abstract}

PACS numbers: 71.15.Pd, 07.05.Tp, 61.20.Ja, 72.20.Jv

The electrostatic interaction between two point charges in a medium with uniform dielectric constant $\epsilon_{0}$ varies as $e_{1} e_{2} / 4 \pi \epsilon_{0} r$. The large numerical value of this energy together with its long range are such that it is very often the most costly component in the simulation of charged condensed matter systems. Naive evaluation of the electrostatic energies in molecular dynamics and Monte-Carlo algorithms leads to inner loops where the summation over all pairs takes a time which scales as $O\left(N^{2}\right)$ for a single step in which all $N$ particles are updated.

Many methods are used to improve this poor scaling: The optimized Ewald algorithm splits the summation between real and Fourier space and has a complexity of $O\left(N^{3 / 2}\right)$ [1, 2]. By interpolation of the charge distribution onto a grid, fast Fourier transform methods allow a scaling in $O(N \log (N))$ [3]. Finally, a popular method in very large simulations is an expansion of the charge distribution using hierarchical multipoles [4, 5]. The asymptotic improvement in efficiency comes, however, with great increases in the complexity of the coding, especially when distributed on multiprocessor computers. The numerical prefactors in these scaling laws are uncomfortably high: Despite the great effort put into optimizing the electrostatic loop, it is found that in the simulation of a large biomolecule (with $N \sim 10^{5}$ ) the great majority of the CPU time is still used in the Coulomb loop [6] in even the most sophisticated numerical codes. Most of these "fast" methods can only be used efficiently in molecular dynamics simulations; there are many occasions where one would like to perform efficient Monte-Carlo simulations due to the stability and simplicity of the method.

The classical methods for treating charged systems have another disadvantage, their inability to treat systems with inhomogeneous dielectric constants. Dielectric inhomogeneities have drastic effects on material properties. For instance the dielectric contrast between water and the core of proteins leads to expulsion of counter-ions from a $3 A$ thick hydration layer [7]. To treat charging effects in proteins quite arbitrary, uncontrolled approximations are made [8] on effective electrostatic interactions in the vicinity of a protein in order to reduce interactions to effective pair-wise additive potentials. Similarly much work [9] has been performed on the phase structure of charged synthetic polymers while neglecting the large dielectric contrasts between water based solvents and oily backbone structures which are surely important in the discussion of the stability of the necklace structures predicted in these systems. At present the most promising algorithms are based on the non-local Marcus energy functional [10, 11].

This letter introduces a local algorithm with a propagating field $\mathbf{E}$ with purely local dynamics on an interpolating grid; it has a complexity in $O(N)$ and is elementary to implement. In contrast to conventional grid methods we do not solve for all the field variables at each integration step; we let the field evolve with its own intrinsic dynamics. We were motivated by the observation that Maxwell's equations, which are local, produce Coulomb interactions due to the propagation of a vectorial field. These dynamic equations are, as we shall see, not the only dynamic way of generating the Coulomb interaction. Our method allows a direct, local implementation of dielectric inhomogeneities. As a demonstration of the method we present an explicit implementation of a local Monte-Carlo algorithm for a charged lattice gas. We note that techniques which interpolate charge degrees of freedom onto a lattice are already very well understood; they form part of standard packages such as Amber [3].

We proceed by showing that the Coulomb interaction can be derived from a constrained variational problem. We then show that the constraint equations are solved locally if we allow electric fields which have both gradient and rotational degrees of freedom. This freedom can be used to produced a local Monte-Carlo algorithm. Finally we present a numerical verification of the method.

The energy of a system of charged particles in a uniform dielectric background is expressed as a function of the electric field $\mathbf{E}$

$$
\mathcal{U}=\epsilon_{0} \int \frac{\mathbf{E}^{2}}{2} \mathrm{~d}^{3} \mathbf{r}
$$

where the electric field is constrained by Gauss's law

$$
\operatorname{div} \mathbf{E}-\rho / \epsilon_{0}=0
$$

It is known from classical electrostatics that one solution of equation (2) is given by $\mathbf{E}=-\operatorname{grad} \phi$ so that $\nabla^{2} \phi=-\rho / \epsilon_{0}$. The general solution to the constraint eq. (2) is thus

$$
\mathbf{E}=-\operatorname{grad} \phi+\operatorname{curl} \mathbf{Q}
$$


where $\phi$ is unique to within an additive constant and $\mathbf{Q}$ arbitrary. In Fourier space the electric field can be expressed as $\mathbf{E}(\mathbf{k})=-i \mathbf{k} \phi+i \mathbf{k} \wedge \mathbf{Q}$. The second term of this expression is perpendicular to $\mathbf{k}$ so that there are two physical degrees of freedom in the $\mathbf{Q}$ field, corresponding to two independent polarization states. We can consider that the field is due to a static potential plus transverse photons.

Let us study the stationary states to the variational problem posed by eq. (1) and eq. (2) by using a Lagrange multiplier, with the functional

$$
\mathcal{A}=\int\left[\epsilon_{0} \frac{\mathbf{E}^{2}}{2}-\lambda(\mathbf{r})\left(\epsilon_{0} \operatorname{div} \mathbf{E}-\rho\right)\right] \mathrm{d}^{3} \mathbf{r},
$$

implying that $\mathbf{E}+\operatorname{grad} \lambda=0$. The Lagrange multiplier is identical to the static electrostatic potential, $\phi$ and the minimum energy is $\mathcal{U}_{\text {Coulomb }}=\frac{\epsilon_{0}}{2} \int(\operatorname{grad} \phi)^{2} \mathrm{~d}^{3} \mathbf{r}$. Consider the energy eq. (1) for an arbitrary $\mathbf{E}$ satisfying the constraints then

$$
\mathcal{U}=\frac{\epsilon_{0}}{2} \int\left[(\operatorname{grad} \phi)^{2}+(\operatorname{curl} \mathbf{Q})^{2}\right] \mathrm{d}^{3} \mathbf{r}
$$

Cross terms vanish, as is shown by integrating by parts.

We now turn to the statistical mechanics of a field with the energy of eq. (1) constrained by Gauss's law. We do not impose that the electric field is calculated from a potential. The partition function of a fixed set of charges in presence of a fluctuating field $\mathbf{E}$ is given by

$$
\mathcal{Z}(\{\mathbf{r}\})=\int \mathcal{D} \mathbf{E} e^{-\int \frac{\beta \epsilon_{0} \mathbf{E}^{2}}{2} \mathrm{~d}^{3} \mathbf{r}} \prod_{\mathbf{r}} \delta\left(\operatorname{div} \mathbf{E}-\rho(\mathbf{r}) / \epsilon_{0}\right)
$$

The argument $\{\mathbf{r}\}$ denotes the fact that the integral over the particle positions has not yet been performed. There are two ways of treating this equation. Either one introduces an integral representation of the delta function or one notes that the integral over the field $\mathbf{E}$ decomposes into a (unique) gradient term and a (non unique) rotation so that

$$
\mathcal{Z}(\{\mathbf{r}\})=e^{-\frac{\beta \epsilon_{0}}{2} \int(\operatorname{grad} \phi)^{2} \mathrm{~d}^{3} \mathbf{r}} \int \mathcal{D} \mathbf{E}_{t} e^{-\frac{\beta \epsilon_{0}}{2} \int \mathbf{E}^{2} \mathrm{~d}^{3} \mathbf{r}}
$$

where $\mathcal{D} \mathbf{E}_{t}=\prod_{\mathbf{r}} \delta(\operatorname{div} \mathbf{E}) \mathcal{D} \mathbf{E}$ performs the summation over all the rotational degrees of freedom of the field described by the potential Q. All the dependence on the particle positions is in the prefactor characterized by the electrostatic potential $\phi$ found by solving Poisson's equation. This prefactor gives the Coulomb interaction between the particles. The remaining integral is independent of the positions of the charges; rather remarkably integration over the full set of fields allowed by the constraint multiplies the standard partition function by a simple constant. This extra factor in the partition function can be ignored.

In the presence of non-uniform dielectric media one proceeds in a similar manner with the energy

$$
\mathcal{U}=\int \frac{\mathbf{D}^{2}}{2 \epsilon(\mathbf{r})} \mathrm{d}^{3} \mathbf{r}
$$

and the constraint $\operatorname{div} \mathbf{D}-\rho=0$. We deduce that the displacement is given by $\mathbf{D}=-\epsilon \operatorname{grad} \phi+\operatorname{curl} \mathbf{Q}$ with $\operatorname{div}(\epsilon \operatorname{grad} \phi)=-\rho$ and

$$
\mathcal{Z}(\{\mathbf{r}\})=e^{-\frac{\beta}{2} \int \epsilon(\mathbf{r})(\operatorname{grad} \phi)^{2} \mathrm{~d}^{3} \mathbf{r}} \int \mathcal{D} \mathbf{E}_{t} e^{-\int \frac{\beta}{2 \epsilon(\mathbf{r})} \mathbf{E}^{2} \mathrm{~d}^{3} \mathbf{r}}
$$

so that

$$
\mathcal{Z}(\{\mathbf{r}\})=\mathcal{Z}_{\text {Coulomb }}(\{\mathbf{r}\}) \mathcal{Z}_{\text {fluct }}(\{\mathbf{r}\})
$$

This time the normalization is a function of the distribution of dielectric inhomogeneities. When implemented in inhomogeneous media our treatment leads to potentials which are the sum of the Coulomb and a fluctuation potential [12] which varies as $1 / r^{6}$ for two widely separated particles. This term comes from thermally driven dipole-dipole interactions: Fluctuations in the field produce an inhomogeneous polarization, $\mathbf{P}$, of the dielectric background. This produces an equivalent charge density of - div $\mathbf{P}$ which interacts via Coulomb's law. Such fluctuation potentials are to be expected from the Lifshitz theory of dielectrics.

We now propose a lattice version of the above equations suitable for numerically studying the thermodynamics of charged systems. The trick is to use the arbitrary vector potential $\mathbf{Q}$ to simplify the calculation of the updated fields after the motion of a charged particle. We need, also, to sum over all rotational degrees of freedom of the field in order to calculate statistical weights from the partition function eq. (6).
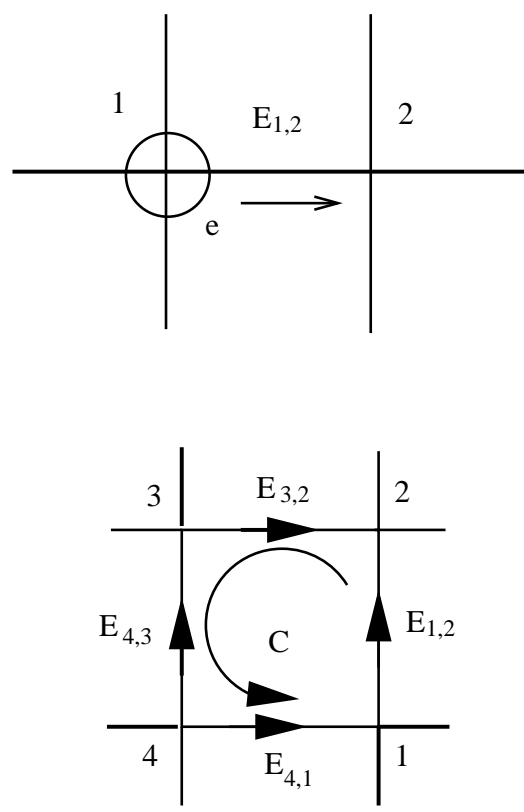

FIG. 1: Top: A charged particle is present on the left lattice point. If the particle is transfered to the right lattice point the constraints are still satisfied if $E_{1,2}$ is modified to $E_{1,2}-e / \epsilon_{0}$ on the connecting link where $e$ is the charge of the particle. Bottom: The four fields associated with a single plaquette, $C$, are modified by a rotational motion.

The observation that we use in order to implement a local 
algorithm is that the constraint equation, eq. (2) can be updated locally in a system in which charge is conserved. We firstly reinterpret the constraint in terms of Faraday's concept of conserved electric flux: Consider, fig. 1 (top), a network where the charges are confined to the vertices $\{i\}$ and the field is associated with links between two sites, $\{i, j\}$. Around each lattice point we imagine a cube and write the constraint in integral form $\int \mathbf{E} . d \mathbf{S}=e_{i} / \epsilon_{0}$. The integral is over the surface of the cube, $e_{i}$ is the enclosed charged at the site. We use the notation $E_{1,2}$ to denote the total flux leaving 1 towards 2; clearly $E_{1,2}=-E_{2,1}$.

The discretized version of the integral constraint is $\sum_{j} E_{i, j}=e_{i} / \epsilon_{0}$ The discretized energy is given by

$$
\mathcal{U}=\frac{\epsilon_{0}}{2} \sum_{\text {links }} E_{i, j}^{2}
$$

Here and in what follows we assume that the lattice spacing is unity.

Start with a system where the constraint is satisfied, fig. 1 (top), and displace a charge, $e$, situated on the leftmost lattice site, 1 , to the rightmost site, 2 . The constraint is again satisfied at both sites if the field associated with the connecting link is updated according to the rule $E_{1,2} \rightarrow E_{1,2}-e / \epsilon_{0}$. This is our Monte-Carlo move for the particles, involving a correlated update of a single charge and the field on the link connecting two sites. To update the field configurations fig. 10 (bottom) we update all the field values of a single plaquette while conserving the constraint at each vertex. In fig. 11 (bottom) $E_{1,2}$ and $E_{4,1}$ increase by an increment $\Delta$ whereas $E_{4,3}$ and $E_{3,2}$ decrease by $\Delta$ so that at each vertex the sum of the entering and leaving fields is constant. It is this last update that performs the integration over all the rotational degrees of freedom in the $\mathbf{E}$ field.

The two moves are not quite sufficient to equilibrate a system with periodic boundary conditions in all situations. This problem is linked with the solution $\phi=-\overline{\mathbf{E}} \cdot \mathbf{r}$ or $\mathbf{E}=\overline{\mathbf{E}}$ of the Laplace equation on a torus where $\overline{\mathbf{E}}$ is an arbitrary constant vector. Motion of the charges generates fluctuations in $\overline{\mathbf{E}}$, while updates such as those in fig. 11(bottom) preserve $\overline{\mathbf{E}}$; similar phenomena also occur with Maxwell's equations. In order to be absolutely sure that the algorithm is ergodic we introduced a third possible Monte-Carlo step which consists of a shift in $\overline{\mathbf{E}}$. By keeping track of the evolution of $\overline{\mathbf{E}}$ as the particles move this last update can be efficiently implemented without destroying the $O(N)$ scaling of the algorithm. In the largest systems fluctuations in this single mode should give a small contribution to the thermodynamics if the initial condition is typical; in such cases this update can be eliminated.

We have performed two initial verifications of the algorithm. Firstly we randomly placed four positive and four negative charged particles on a $4 \times 4 \times 4$ lattice with periodic boundary conditions. We performed field updates using the Metropolis algorithm at zero temperature in order to anneal the field E. We then solved for the electric fields using a standard linear algebra package. The results were identical to within numerical errors. Annealing $\overline{\mathbf{E}}$ was crucial in order to get agreement between the two methods with frozen charges. A second check was then performed with two different implementations of the Metropolis algorithm now run at several finite temperatures: 36 mutually avoiding, charged particles were distributed in a $6 \times 6 \times 6$ cube with periodic boundary conditions. The dielectric constant was uniform. In the first simulation the linear solver was used to calculate the exact interaction energy of the charged particles at each Monte-Carlo step. In the second simulation we implemented the above local algorithm. For the two methods we then compared the static structure factor for charge-charge correlations, finding good agreement.

We verified the efficiency of the algorithm by determining the autocorrelation time of the slowest density and charge modes in a cube of dimension $L$ using the method described in [13], fig. (2]. We plot the relaxation time in Monte-Carlo sweeps; during each sweep an average of one Monte-Carlo trial is performed for each degree of freedom in the simulation. As expected in a Monte-Carlo algorithm dominated by diffusive motion the slowest mode relaxes in a time which varies as $L^{2}$. There is no anomalous or critical slowing down due to the coupling between the particles and the electric field. The saturation of the relaxation time for charge fluctuations at large $L$ is a consequence of screening; charge fluctuations relax by diffusion over the Debye length.

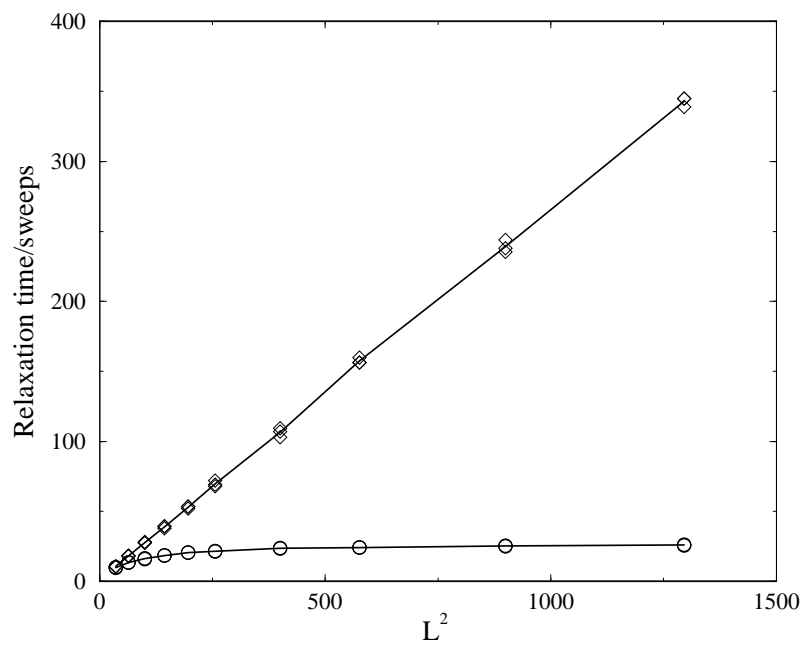

FIG. 2: Relaxation times in Monte-Carlo sweeps for the densitydensity (top) correlation function and the charge-charge (bottom) correlation function measured for the mode $\frac{2 \pi}{L}(1,0,0)$ plotted as a function of $L^{2}$. The top curve shows scaling compatible with simple diffusion. The bottom curve saturates for large systems sizes. The multiple points for each $L$ correspond to three independent determinations of the relaxation time. System sizes between $L=6$ and $L=36$ with $L^{3} / 4$ charged particles. $6 \times 10^{5}$ sweeps per simulation for a total simulation time of three days on a AMD Athlon computer.

We have shown that the thermodynamics of charged systems can be simulated locally by introducing a propagating vector field so that particles interact via retarded, diffusing 
fields. The dynamic properties of the system are strongly modified but by construction thermodynamics is an invariant of the propagation dynamics. Our treatment of the dynamics of the field $\mathbf{E}$ is similar to the Coulomb or radiation gauge in classical electrodynamics: Normally one writes that

$$
\mathbf{E}=-\operatorname{grad} \phi-\frac{\partial \mathbf{A}}{\partial t}
$$

where in the Coulomb gauge $\operatorname{div} \mathbf{A}=0$. There is a rather close analogy between our dynamic scheme of solving for the constraint equations with certain methods of quantization in the Coulomb gauge: In Dirac's quantization of the electrodynamic field Gauss's law is a weak identity [14] dependent on the choice of the initial wave function. In our simulation Gauss's law is the result of a restricted choice of possible moves in the Monte-Carlo algorithm together with a special initial condition.

What are the advantages of the present method over direct integration of Maxwell's equations which are also an example of an $O(N)$ algorithm [18]? Monte-Carlo algorithms are particularly easy to implement and have good stability with large step sizes. In addition, we have checked that the fast equilibration of the electric degrees of freedom in fig. (2) allows one to perform field updates far more rarely than particle updates leading to additional acceleration of the algorithm. Such multiple time step ideas have been applied to conventional electrostatic solvers but in molecular dynamics are sometimes prone to numerical instabilities [6].

In the implementation of the algorithm we were inspired by recent work on hydrodynamic interactions (varying as $1 / r$ ) via a Lattice-Boltzmann algorithm in simulations of polymer solutions [15]. Another analogous problem to ours has been treated by Car and Parrinello who have shown [16] that introduction of a fictitious dynamics leads to much improved efficiencies in solving for constraints; our work differs in that the constraint of Gauss's law is solved exactly at each simulation step whereas the Car-Parrinello algorithm leads to a simulated annealing solution of the constraint equations (2).

Finally let us note that other functionals do exist for the electric potential in the presence of sources [17]. In particular the functional

$$
\int\left[\frac{\epsilon_{0}}{2}(\nabla \phi)^{2}-\rho \phi\right] \mathrm{d}^{3} \mathbf{r}
$$

seems, at first sight particularly simple and attractive. Unfortunately, the minimum of this functional is minus the correct electrostatic energy. It can not be used as a functional for both the field evolution and the particle motion. Application of the algorithm to large atomistic systems remain to be tested, but our method provides an alternative to existing treatments of Coulomb interactions.

We would like to thank R. Everaers for many crucial discussions in the formulation of this work, in particular for his remarks on the importance of dielectric effects.

[1] J. V. L. Beckers, C. P. Lowe, and S. W. de Leeuw, Molecular Simulation 20, 269 (1988)

[2] J. W. Perram, H. G. Petersen, and S. W. de Leeuw, Molecular Phys. 65, 875 (1988).

[3] E. Essmann, L. Perera, M. L. Berkowitz, T. Darden, H. Lee, and L. G. Pedersen, J. Chem. Phys. 103, 8577 (1995).

[4] J. E. Barnes and P. Hut, Nature 324, 446 (1986).

[5] L. Greengard and V. Rokhlin, J. Comput. Phys. 73, 325 (1987).

[6] T. Schlick, R. D. Skeel, A. T. Brunger, L. V. Kalé, J. A. Board Jr., J. Hermans, and K. Schulten, J. Comp. Phys. 151, 9 (1999).

[7] M. F. Perutz, Science 206, 1187 (1979).

[8] T. Solmajer and E. L. Mehler, Protein Engin. 4, 911 (1991).

[9] Y. Kantor, H. Li, and M. Kardar, Phys. Rev. Let.t 69, 61 (1992).

[10] R. A. Marcus, J. Chem. Phys. 24, 966 (1956).

[11] M. Marchi, D. Borgis, N. Levy, and P. Ballone, J. Chem. Phys 114, 4377 (2001).

[12] L. D. Landau and E. M. Lifshitz, Statistical Physics (Butterworth-Heinemann, 1999).

[13] A. C. Maggs, cond-mat/0111272, to appear J. Chem. Phys. (2001).

[14] P. A. M. Dirac, The Principles of Quantum Mechanics (Oxford University Press, 2000).

[15] P. Ahlrichs, R. Everaers, and B. Dünweg, Phys. Rev. E 64, 040501(R) (2001).

[16] R. Car and M. Parrinello, Phys. Rev. Let. 55, 2471 (1985).

[17] J. D. Jackson, Classical Electrodynamics (Wiley, 1999).

[18] We thank the reviewer for this remark 